\title{
Probing light Yukawa couplings in Higgs pair production
}

\author{
Lina Alasfar* \\ Humboldt-Universität zu Berlin, Institut für Physik, Newtonstr. 15, 12489 Berlin, \\ Germany. \\ E-mail: lina.alasfarephysik.hu-berlin.de

\section{Ramona Gröber} \\ Humboldt-Universität zu Berlin, Institut für Physik, Newtonstr. 15, 12489 Berlin, \\ Germany. \\ Dipartimento di Fisica e Astronomia "G. Galilei", Università di Padova, Italy. \\ Istituto Nazionale Fisica Nucleare, Sezione di Padova, I-35131 Padova, Italy. \\ E-mail: ramona.groeber@pd.infn.it
}

\begin{abstract}
One of the puzzles of the SM is the large hierarchy between the Yukawa couplings of different flavours. Yukawa couplings of the first and the second generation are constrained only very weakly so far. However, one can obtain large deviations in the Yukawa couplings in several New Physics (NP) models, such as e.g. models with new vector-like quarks, or new Higgs bosons that couple naturally to individual fermion families.

In this work, we investigate the potential bounds on the NP Higgs Yukawa couplings modification $\kappa_{f}$ for light quarks from double-Higgs at the LHC, starting from a model independent formalism. We have looked at the two Higgs boson final state $b \bar{b} \gamma \gamma$, and the relevant experimental cuts to reduce backgrounds and estimated the potential exclusion bounds for $\kappa_{f}$. We have considered both linear and non-linear effective field theory for the Higgs light quark coupling modifications.
\end{abstract}

European Physical Society Conference on High Energy Physics - EPS-HEP2019 -

10-17 July, 2019

Ghent, Belgium

Report Number : HU-EP-19/29.

\footnotetext{
${ }^{*}$ Speaker.
} 


\section{Effective field theory of light Yukawa couplings}

In the Standard Model (SM), the Higgs doublet $\phi$ couples to the quarks via a Yukawa interaction term

$$
\mathscr{L}_{y}=-y_{i j}^{u} \bar{Q}_{L}^{i} \tilde{\phi} u_{R}^{j}-y_{i j}^{d} \bar{Q}_{L}^{i} \phi d_{R}^{j}+\text { h.c. },
$$

with $\tilde{\phi}=i \sigma_{2} \phi^{*}, \sigma_{2}$ is the second Pauli matrix, $Q_{L}^{i}$ the left-handed $S U(2)$ quark doublet of the $i$-th generation and $u_{R}^{j}$ and $d_{R}^{j}$ the right-handed up- and down-type fields of the $j$-th generation, respectively.

It is possible to modify this coupling via a dimension 6 operator in the SM effective field theory formalism (SMEFT) with a UV cutoff scale $\Lambda$

$$
\Delta \mathscr{L}_{y}=\frac{\phi^{\dagger} \phi}{\Lambda^{2}}\left(c_{i j}^{u} \bar{Q}_{L}^{i} \tilde{\phi} u_{R}^{j}+c_{i j}^{d} \bar{Q}_{L}^{i} \phi d_{R}^{j}+\text { h.c. }\right) .
$$

The resulting mass matrices can be diagonalised by means of a bi-unitary transformation $V_{L / R}^{q}$ in order to recover the measured quark masses. The Yukawa couplings $(h \bar{q} q)$ will be modified by this new operator. Moreover, a new coupling between quark anti-quark pair and two Higgs bosons $h h \bar{q} q$ appears with coupling constant

$$
g_{h \bar{q}_{i} q_{j}}: \frac{m_{q_{i}}}{v} \delta_{i j}-\frac{v^{2}}{\Lambda^{2}} \frac{\tilde{c}_{i j}^{q}}{\sqrt{2}}, \quad \quad g_{h h \bar{q}_{i} q_{j}}: \quad-\frac{3}{2 \sqrt{2}} \frac{v}{\Lambda^{2}} \tilde{c}_{i j}^{q},
$$

where

$$
\tilde{c}_{i j}^{q}=\left(V_{L}^{q}\right)_{n i}^{*} c_{n m}^{q}\left(V_{R}^{q}\right)_{m j}, \quad \text { with } \quad q=u, d,
$$

and $V_{L / R}^{q}$ denote the transformation matrices from current to mass eigenstates.

It has been shown in $[1,2,3]$ that it is possible to introduce large modifications to the Yukawa couplings without flavour-changing neutral currents (FCNCs) at tree-level, which strongly constrain off-diagonal couplings [4], and without assuming minimal flavour violation (MFV) [5]. Very little is known though about the flavour-diagonal Higgs coupling to light quarks, as seen from the weak bounds on these coupling modifications, $c f$. ref. [6]. Therefore, we only restrict ourselves to varying the diagonal light quark Higgs couplings, writing

$$
g_{h \bar{q}_{i} q_{i}}=\kappa_{q} g_{h \bar{q}_{i} q_{i}}^{\mathrm{SM}}, \quad \quad g_{h h \bar{q}_{i} q_{i}}=-\frac{3}{2} \frac{1-\kappa_{q}}{v} g_{h \bar{q}_{i} q_{i}}^{\mathrm{SM}}
$$

Here, we have abused the $\kappa$-formalism notation that is used heavily in experimental searches.

Another way to modify the Yukawa couplings in a model-independent way is via the electroweak chiral Lagrangian [7]. Using the chiral Lagrangian by taking the 0th chiral mode and restricting ourselves to the flavour diagonal couplings we get

$$
-\mathscr{L}=\bar{q}_{L} \frac{m_{q}}{v}\left(v+c_{q} h+\frac{c_{q q}}{v} h^{2}+\ldots\right) q_{R}+\text { h.c. }
$$

Thus, in this formalism, the Yukawa and $h h \bar{q} q$ couplings are uncorrelated, making the Higgs pair production the most accessible process to test the potential correlation between the Higgs-light quark couplings. 

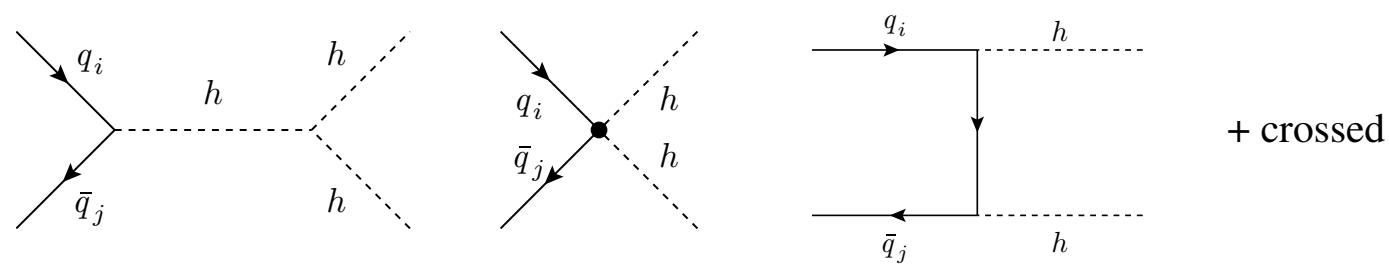

Figure 1: Feynman diagrams for the qqA Higgs pair production.

\section{Higgs pair production with modified Yukawa couplings}

In the SM, the production of Higgs pairs in hadron colliders (such as the LHC) is dominated by gluon-gluon fusion (ggF) which at leading order (LO) is $\sim 21 \mathrm{fb}$.

Higher order QCD corrections to this process have been computed up to order $\alpha_{s}^{4}$ [8]. For the inclusive cross section the LO calculation can be corrected by a K-factor,

$$
K=\frac{\sigma_{N N L O}}{\sigma_{L O}}, \quad K_{14 \mathrm{TeV}}=1.72
$$

In our analysis, we use the state-of-the-art calculation of the ggF inclusive cross section at 14 $\mathrm{TeV}[9] \sigma_{N N L O}^{\mathrm{SM}}=36.69_{-2.57}^{+1.99} \mathrm{fb}$.,

However, if the light quark Yukawa couplings are significantly increased, the $\mathrm{ggF}$ process will become subdominant compared to the quark anti-quark annihilation (qqA), due to the abundance of light quarks in the proton. This happens if the coupling modifiers are of order $\kappa_{c}^{q q A=g g F} \sim 5$, $\kappa_{s}^{q q A=g g F} \sim 10$ and $\kappa_{u}^{q q A=g g F} \sim \kappa_{d}^{q q A=g g F} \sim 10^{3}$. Those values are not yet excluded by current measurements. The qqA Higgs pair production is calculated from the diagrams shown in fig. 1. up to NLO QCD, see [3]. The shape difference between the normalized ggF and qqA dominant distributions is used in the analysis to extract sensitivity limits on modifications of light quark Yukawa couplings.

\section{Phenomenological analysis}

In order to estimate the sensitivity of the high-luminosity LHC (HL-LHC) for the light quark scalings, we have used the profile likelihood method to estimate the signal strength $\mu$, defined as (for general resonance $R$ production)

$$
\mu=\frac{N_{\text {expec }}}{N_{\text {expec }}^{S M}}, \quad \text { with } \quad N_{\text {expec }}=\sigma(p p \rightarrow R) \mathscr{B}(R \rightarrow X) L \varepsilon_{\mathrm{SEL}},
$$

where $\sigma(p p \rightarrow R) \mathscr{B}(R \rightarrow X)$ is the production cross section of $R$ times the branching fraction for the final state $X, L=3 \mathrm{ab}^{-1}$ the integrated luminosity of the HL-LHC, and $\varepsilon_{\mathrm{SEL}}$ is the experimental selection.

In our analysis we have chosen the two Higgs final state $h h \rightarrow b \bar{b} \gamma \gamma$ due to its high potential of reconstruction at the HL-LHC [10, 11]. We have modified the FORTRAN programme HDECAY [12] to obtain the Higgs boson branching ratios, including state-of-the art QCD corrections and the light fermion loops and decay channels. 
The Higgs pair production and decays were implemented into Pythia 6.4 for parton showering. Then the events were analysed according to [13] in order to estimate the selection efficiency. In addition to $b$-tagging, we have used charm mistagging probability of $b$-jets combined with $c$-tagging working points for CMS [14] and ATLAS [15], as developed in [16, 17] in order to constrain $\kappa_{c}$ and $\kappa_{s}$. This will allow us to put bounds on the second generation quark Yukawa couplings, while otherwise no sensitivity can be obtained, due to the fact that the lowering of the branching fraction of the final state $h h \rightarrow b \bar{b} \gamma \gamma$ is larger than the cross-section enhancement. This method will allow us to probe the final state $h h \rightarrow c \bar{c} \gamma \gamma$ using the current $c$-tagging techniques [18, 19] or the improved ones after the ATLAS HL-LHC upgrade [20,21], thus in total improving the expected sensitivity for the second generation couplings.

\section{Results}

Using a likelihood fit for estimating $\mu$, assuming that SM Higgs pair production is the null hypothesis, then preforming a scan over $\kappa_{u}$ and $\kappa_{d}$, we obtain the $68 \%$ and $95 \%$ CL sensitivity likelihood contours for the HL-LHC shown in fig. 2.

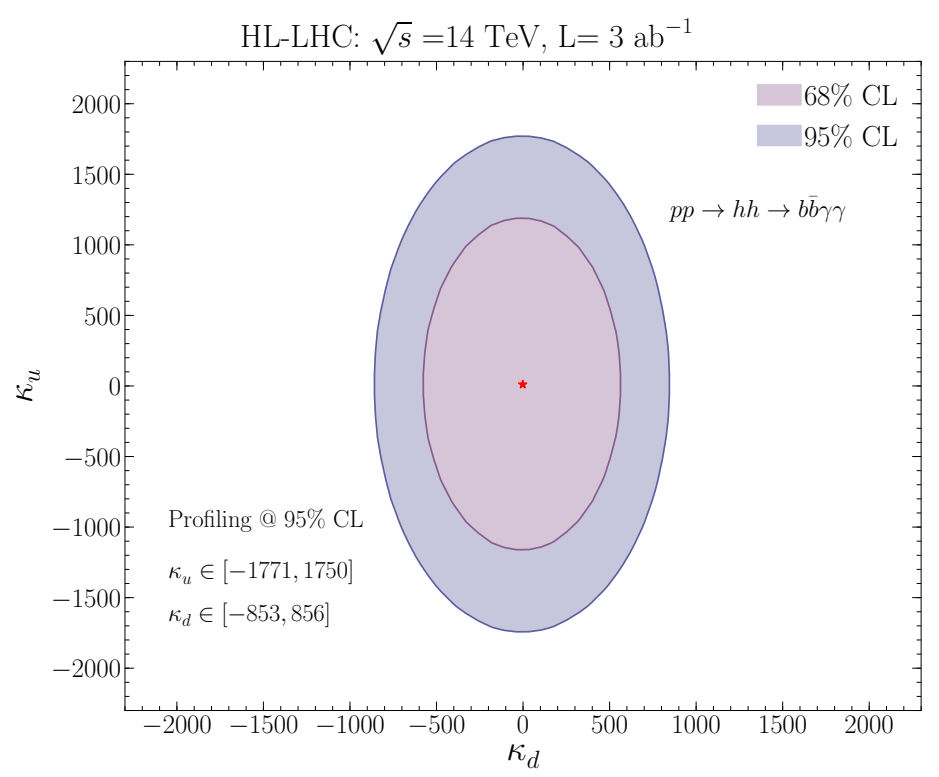

Figure 2: The expected sensitivity likelihood contours at $68 \%$ and 95\% CL of the HL-LHC for the first generation Yukawa coupling scalings.

We show now results for non-linear EFT Wilson coefficients $c_{q}$ and $c_{q q}$ of the chiral Lagrangian of eq. (1.6) by scanning over them separately in order to obtain the sensitivity bounds on the non-linear EFT illustrated in fig. 3.

For the linear EFT hypothesis, it was not possible to construct sensitivity bounds using only the final state with $b$-quarks for the second generation quarks as discussed before. We hence directly include into fig. 4 final states with $c$-quarks, which depends on the $c$-tagging working points as discussed also in $[3,16,22]$. The expected sensitivity limits for the charm quark Yukawa modifications are an improvement compared to the current direct bounds and prospects for the HL-LHC, see [16, 22]. 

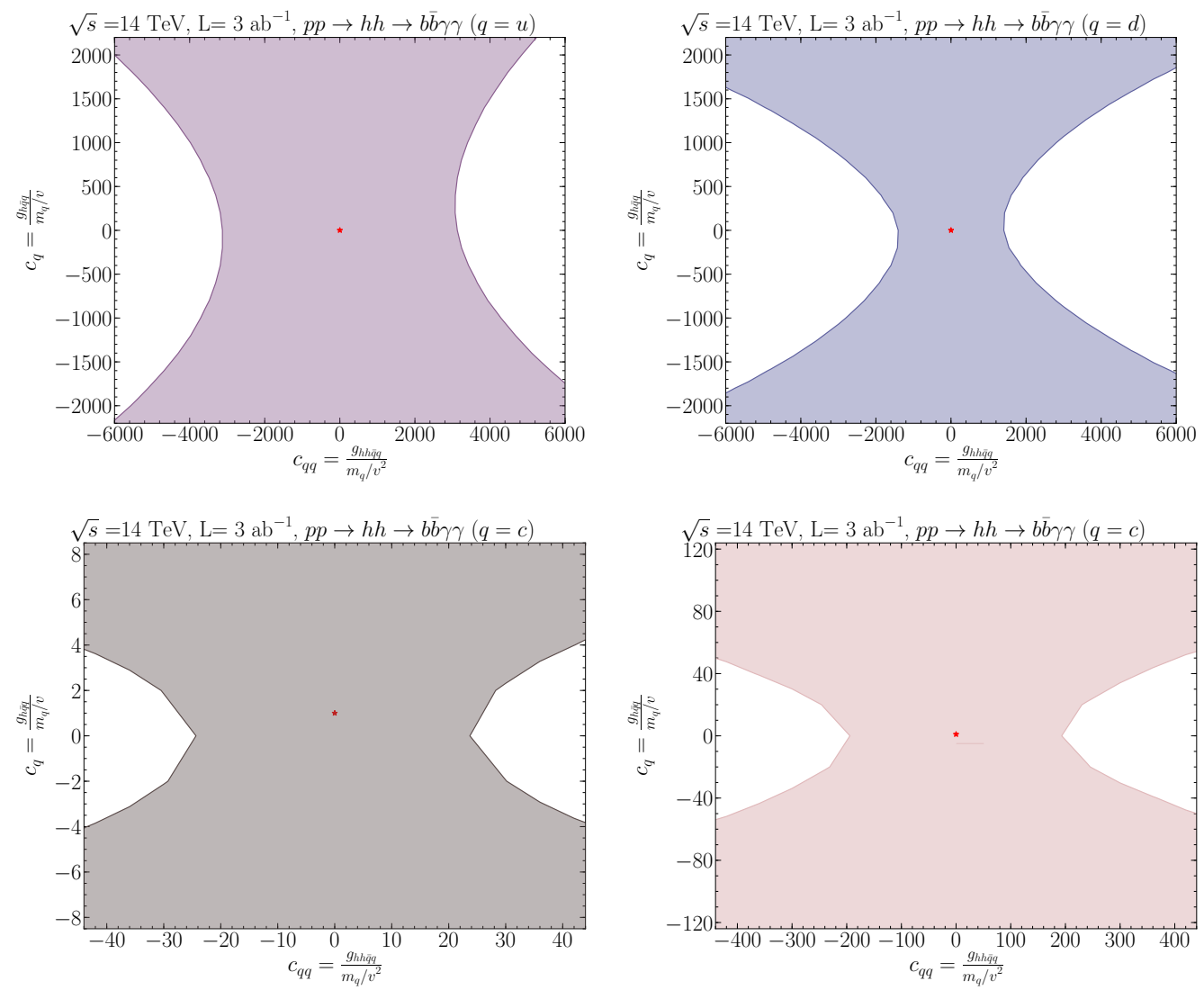

Figure 3: 95\% CL likelihood contours for the non-linear EFT coefficients $c_{q q}$ and $c_{q}$ for up (upper left), down (upper right), charm (lower left) and strange quarks (lower right).

\section{Conclusion}

Searching for the production of Higgs pairs $(h h)$ provides a great insight into the least understood Higgs couplings, the trilinear Higgs self-coupling and the light quark Yukawa couplings. As we showed, it is possible to set prospective model-independent bounds on the light quark Yukawa couplings which are comparable to the prospects from a global fit making use of the Higgs pair production process. The expected sensitivity is $\left|\kappa_{u}\right| \lesssim 1170$ and $\left|\kappa_{d}\right| \lesssim 850, c f$. fig. 2.

Moreover, with mixed $b$ - and $c$-tagging working points, the Higgs pair production provides competitive sensitivity for constraining modifications of the charm Yukawa coupling i.e. $\left|\kappa_{c}\right| \lesssim 5$ and $\left|\kappa_{s}\right| \lesssim 100$, cf. fig. 4 , where the first prospective limit is comparable to the prospects from charm tagging in the $V h$ channel [16]. Furthermore, it turns out that the process is in particularly sensitive to the non-linearities in the light quark-Higgs couplings, so providing a possible insight into the linear or non-linear nature of the Higgs boson.

\section{Acknowledgements}

We thank R. Corral Lopez for collaboration on this topic and the organisers of the conference for a pleasant and stimulating atmosphere during the conference. RG was in parts supported by the 

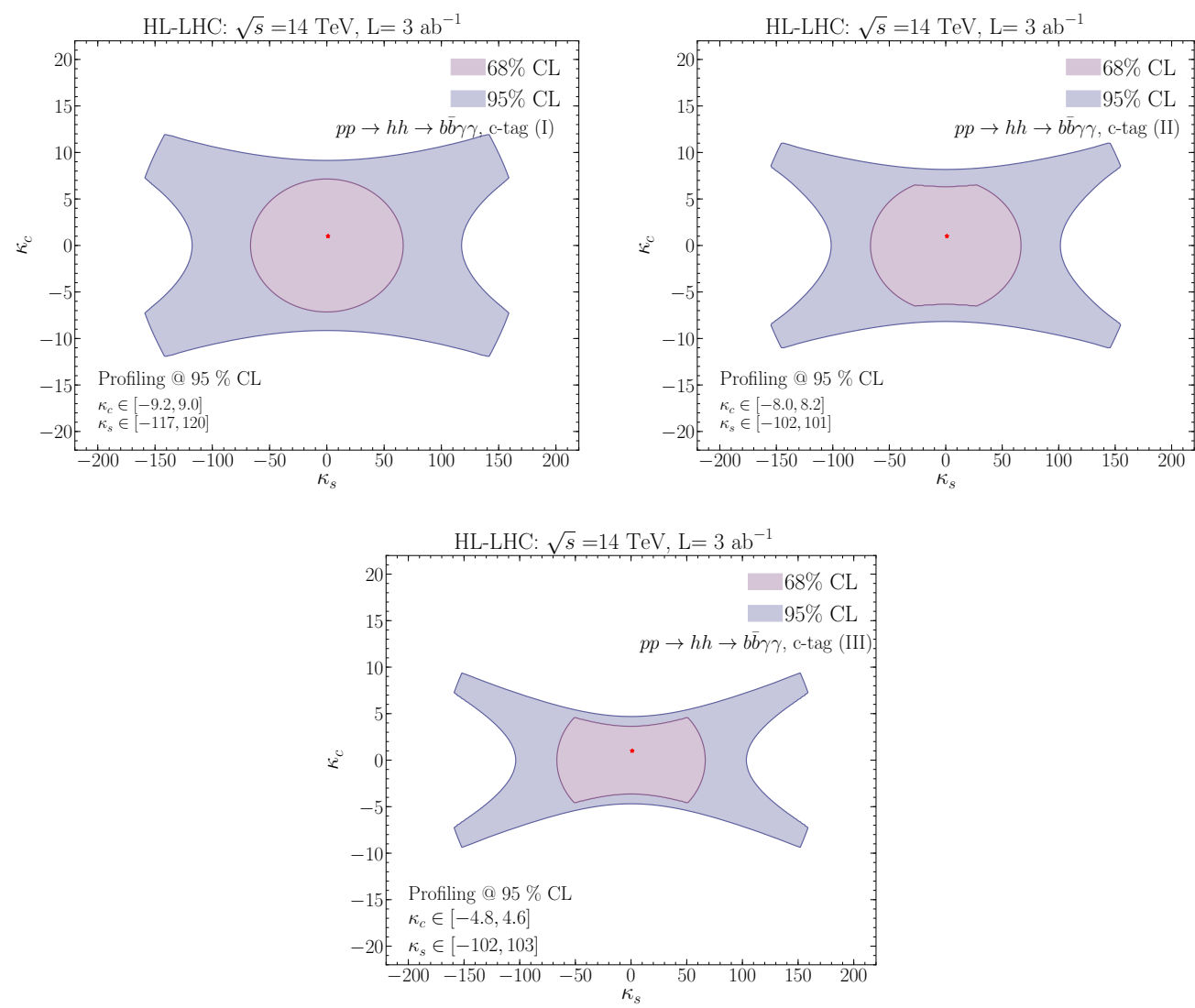

Figure 4: The expected sensitivity likelihood contours at 68\% CL and 95\% CL for an integrated luminosity $L=3 \mathrm{ab}^{-1}$ for modified second generation quark Yukawa couplings, using the c-tagging I (upper pannel, left), II (upper pannel, right) and III (lower pannel) working points, see [3].

"Berliner Chancengleichheitsprogramm".

\section{References}

[1] S. Bar-Shalom and A. Soni, "Universally enhanced light-quarks Yukawa couplings paradigm," Phys. Rev. D98 no. 5, (2018) 055001, arXiv: 1804.02400 [hep-ph] .

[2] D. Egana-Ugrinovic, S. Homiller, and P. Meade, "Aligned and Spontaneous Flavor Violation," Phys. Rev. Lett. 123 no. 3, (2019) 031802, arXiv:1811.00017 [hep-ph] .

[3] L. Alasfar, R. C. Lopez, and R. Gröber, "Probing Higgs couplings to light quarks via Higgs pair production," arXiv:1909.05279 [hep-ph].

[4] G. Blankenburg, J. Ellis, and G. Isidori, "Flavour-Changing Decays of a $125 \mathrm{GeV}$ Higgs-like Particle," Phys. Lett. B712 (2012) 386-390, arXiv:1202.5704 [hep-ph] .

[5] G. D'Ambrosio, G. F. Giudice, G. Isidori, and A. Strumia, "Minimal flavor violation: An Effective field theory approach," Nucl. Phys. B645 (2002) 155-187, arXiv: hep-ph/ 0207036 [hep-ph] .

[6] J. De Blas et al., "Higgs Boson Studies at Future Particle Colliders," arXiv: 1905.03764 [hep-ph]. 
[7] S. R. Coleman, J. Wess, and B. Zumino, "Structure of phenomenological Lagrangians. 1.," Phys. Rev. 177 (1969) 2239-2247.

[8] O. Éboli, G. Marques, S. Novaes, and A. Natale, "Twin higgs-boson production," Physics Letters B 197 no. $\sim 1,(1987) 269-272$.

[9] M. Grazzini, G. Heinrich, S. Jones, S. Kallweit, M. Kerner, J. M. Lindert, and J. Mazzitelli, "Higgs boson pair production at NNLO with top quark mass effects," JHEP 05 (2018) 059, arXiv:1803.02463 [hep-ph].

[10] U. Baur, T. Plehn, and D. L. Rainwater, "Probing the Higgs selfcoupling at hadron colliders using rare decays," Phys. Rev. D69 (2004) 053004, arXiv: hep-ph/ 0310056 [hep-ph] .

[11] J. Baglio, A. Djouadi, R. Gröber, M. M. Mühlleitner, J. Quevillon, and M. Spira, "The measurement of the Higgs self-coupling at the LHC: theoretical status," JHEP 04 (2013) 151,

arXiv:1212.5581 [hep-ph] .

[12] A. Djouadi, J. Kalinowski, and M. Spira, "HDECAY: A Program for Higgs boson decays in the standard model and its supersymmetric extension," Comput. Phys. Commun. 108 (1998) 56-74, arXiv:hep-ph/9704448 [hep-ph].

[13] A. Azatov, R. Contino, G. Panico, and M. Son, "Effective field theory analysis of double Higgs boson production via gluon fusion," Phys. Rev. D92 no. 3, (2015) 035001, arXiv:1502.00539 [hep-ph] .

[14] CMS Collaboration, S. Chatrchyan et al., "Search for the standard model Higgs boson produced in association with a W or a Z boson and decaying to bottom quarks," Phys. Rev. D89 no. 1, (2014) 012003, arXiv:1310.3687 [hep-ex].

[15] ATLAS Collaboration, G. Aad et al., "Search for the $b \bar{b}$ decay of the Standard Model Higgs boson in associated $(W / Z) H$ production with the ATLAS detector," JHEP 01 (2015) 069, arXiv:1409.6212 [hep-ex].

[16] G. Perez, Y. Soreq, E. Stamou, and K. Tobioka, "Constraining the charm Yukawa and Higgs-quark coupling universality," Phys. Rev. D92 no. 3, (2015) 033016, arXiv:1503.00290 [hep-ph].

[17] D. Kim and M. Park, "Enhancement of new physics signal sensitivity with mistagged charm quarks," Phys. Lett. B758 (2016) 190-194, arXiv:1507.03990 [hep-ph].

[18] ATLAS Collaboration, G. Aad et al., "Search for Scalar Charm Quark Pair Production in $p p$ Collisions at $\sqrt{s}=8 \mathrm{TeV}$ with the ATLAS Detector," Phys. Rev. Lett. 114 no. 16, (2015) 161801, arXiv:1501.01325 [hep-ex].

[19] ATLAS Collaboration, "Search for single top-quark production via FCNC in strong interaction in $\sqrt{s}=8 \mathrm{TeV}$ ATLAS data," Tech. Rep. ATLAS-CONF-2013-063, CERN, Geneva, 2013. http://cds.cern.ch/record/1562777.

[20] ATLAS Collaboration, M. Capeans, G. Darbo, K. Einsweiller, M. Elsing, T. Flick, M. Garcia-Sciveres, C. Gemme, H. Pernegger, O. Rohne, and R. Vuillermet, “ATLAS Insertable B-Layer Technical Design Report,” Tech. Rep. CERN-LHCC-2010-013. ATLAS-TDR-19, 2010.

[21] ATLAS Collaboration, "Track Reconstruction Performance of the ATLAS Inner Detector at $\sqrt{s}=13$ TeV,” Tech. Rep. ATL-PHYS-PUB-2015-018, CERN, Geneva, 2015.

[22] G. Perez, Y. Soreq, E. Stamou, and K. Tobioka, "Prospects for measuring the Higgs boson coupling to light quarks," Phys. Rev. D93 no. 1, (2016) 013001, arXiv:1505.06689 [hep-ph]. 